\title{
Review Article \\ From Lonely to Resilient through Humanoid Robots: Building a New Framework of Resilience
}

\author{
Johan F. Hoorn (iD) 1,2 \\ ${ }^{1}$ Department of Computing and School of Design, The Hong Kong Polytechnic University, Hong Kong KLN, Hong Kong \\ ${ }^{2}$ Department of Communication Science, Vrije Universiteit, Amsterdam 1081 HV, Netherlands \\ Correspondence should be addressed to Johan F. Hoorn; johan.f.hoorn@polyu.edu.hk
}

Received 15 March 2018; Revised 18 May 2018; Accepted 27 June 2018; Published 19 July 2018

Academic Editor: Shih-Yi Chien

Copyright (c) 2018 Johan F. Hoorn. This is an open access article distributed under the Creative Commons Attribution License, which permits unrestricted use, distribution, and reproduction in any medium, provided the original work is properly cited.

\begin{abstract}
This paper connects different theories and methods from the social sciences and applies them to human-humanoid robot interaction (HHRI) to explain loneliness reduction and the build-up of resilience in older adults through social robots. It allows for userrelated aspects such as age, social connectedness, gender role, personality, and need satisfaction as well as robot-related aspects, particularly coaching behaviors and communication styles. From these scientific considerations, solutions to design challenges are pinpointed, proposing novel interaction schemes that enhance the feeling of support and companionship. This paper also opens the way to conducting empirical research to examine HHRI-related designs, measuring user experience in HHRI, while suggesting applications in HHRI in various settings, such as coaching and eldercare.
\end{abstract}

\section{Introduction}

Loneliness is an increasing societal issue world-wide [1, 2] with severe negative effects on health and wellbeing [3]. An aging population often leads to greater loneliness, which negatively affects public health and quality of life. Loneliness is the tension between a current and a desired situation of social interaction and connectedness [4]. I will attempt the study of resilience or 'flexibility' of older adults to counter loneliness while they are supported by humanoid robots. To this end, a novel theoretical framework of resilience is proposed, which is open to formal modeling and empirical testing.

Note, however, that the longitudinal study proposed in the current paper will take a number of years before completion. Therefore, I want to confront the theoretical framework and envisioned method with the readership before putting great effort into sampling longitudinal data for the wrong theoretical reasons or with a flawed method in place. In other words, the current paper develops hypotheses and proposes how to measure these but does not present actual data, which is work left for the future.

Thus far, interventions that directly address loneliness do not seem to be too successful [5]. This might be because such approaches wish to diminish the negative (loneliness) instead of improve the positive (resilience). It would be worthwhile if people could counter loneliness themselves by enhancing their resilience, which to date is left untried [6].

I would want to characterize personal resilience as the flexibility of an individual to positively deal with hardship. Because resilience is used as somewhat of a container term, the definitions of resilience are rather tentative and the level of data aggregation (individual or group) could sometimes benefit from more determination. Although the notion of resilience seems to be commonly understood, an explanatory model is still wanting and mathematical modeling is practically absent.

Yet, I consider a mathematical account with explanatory power as an important requirement on a robust and predictive theory, which would be the overall scientific aim. In knowing the state of the art, however, formalization, robustness, and predictive value will be more of an ideal than a feasible result of this paper, so I want to do some groundwork instead and prepare for that later effort. For now, my objective is to arrive at more explicit definitions and demarcations, which allow the formulation of an explanatory model that eventually can be tested empirically and implemented in applications of human-humanoid robot interaction (HHRI). 
One of the first things to realize is that resilience is always related to something else. In this case, I wish to study resilience in older adults, who increasingly face the hardship of loneliness [7]. Resilience in combination with loneliness has not been studied before and the main question is how they affect each other: will more resilience mitigate loneliness or will increased loneliness decrease resilience?

Older adults respond well to robots, particularly when they feel lonely [8]. However, Frennert and Östlund [9] express their concerns that a humanoid robot's social role in the lives of older adults often remains implicit, that methods for longitudinal study are wanting, that seniors and robot developers are mutually uninterested in the development of social robots for older adults, and that conceptual clarity in HHRI is missing for matters that concern older adults. Additionally, Frennert and Östlund [9] state that in HHRI often technical determinism takes precedence over the social construction of humanoid robots. In the current paper, I attempt to tackle these issues by exploring how resilience and loneliness are affected by social connectedness and (quality of) relationship [10-12]. Meanwhile, I pose the question whether robots can make up for a degraded social environment, improve resilience in older adults, and in so doing mitigate their loneliness?

Figure 1 provides a general overview of my argument. It may serve as a guideline for the reader and I will refer to it throughout the paper. It assembles all the research questions in relationship to each other. It provides for the conceptualization of resilience as a capacity, a process, and an outcome. It then discusses robot support of the resilience process to ease loneliness as flanked by longitudinal studies into resilience (to be conducted yet), using a particular robotmediated conference-call system.

In the remainder of this paper, I attempt an explanation of the way social robots may positively affect the relation between resilience development and loneliness reduction. I start from the assumption that people are able to recover from loneliness themselves and that the robot merely is a facilitator or instigator of that process. In reducing loneliness indirectly, the progression of resilience complements methods that directly reduce loneliness. From the existing literature, I will argue that, conceptually, resilience is a combination of a capacity, a process, and an outcome. I will pose six research questions (RQ1-6) in relation to a new theoretical framework that helps address those questions. In the end, I also propose a method to test that framework longitudinally with older adults. Note, however, that the test results of the proposed method are pending still. The present paper merely provides a conceptual framework of HHRI and several interaction schemes, addressing the relationship between resilience development and loneliness reduction through humanoid robots.

\section{Resilience}

The overall scientific objective, as stated, is to create a robust and predictive theory of personal resilience. And to get there, the first question would be (Figure 1: RQ1) how the literature defines personal resilience and at what aggregation level?
What factors affect personal resilience? And what would an explanatory model of resilience look like?

In overviewing the literature, studies that can be read as theories of personal resilience cover such diverse topics as clinical problems after disaster and trauma exposure $[13,14]$ but also societal and organizational issues $[15,16]$. Dependent of context, 'resilience' means different things at different levels of aggregation (person, group, and organization). Sometimes it is an outcome or a process, and sometimes it is a personal trait.

In psychosocial intervention, resilience as a process pertains to positive adaptation to misfortune to achieve certain goals $[17,18]$. Goals may be physical (e.g., nourishment, a safe home) or spiritual (e.g., dignity) but do not always have to be achieved completely [19]. Positive adaptation depends on personal traits, moderated by the environment [20, 21]. As an outcome, someone feels more or less resilient [19]. People may show different levels of resilience dependent on age, the stage of life, or the domain (e.g., home or work) [18]. Resilient individuals together form a resilient organization, society, or culture [22].

The apparent conceptual ambiguity about resilience being an outcome, process, or trait may be resolved if we see them as different aspects of the same system (Figure 1). Regarding personality, resilience is the capacity to overcome a crisis situation in a positive way. That capacity may be inborn but also can be learned [23] through a whole range of techniques such as "mindful meditation" and "psychosocial" intervention [24], by giving meaning to suffering and constructing the meaning of life, sense-making, or storytelling [19]. Applying such techniques to achieve higher levels of resilience may be called the resilience process. As an outcome, the level of resilience may fluctuate given certain circumstances. Hence, I will speak of resilience capacity, resilience process, and level of resilience as three related but different theoretical dimensions (Figure 1).

The literature seems to forward two personal and one environmental factor that affect resilience. One personal factor is the ability to successfully interact with the environment to realize one's potential and to achieve one's goals [25]. That could count as an aspect of resilience capacity, the ability to interact. The other personal factor the literature mentions pertains to what can be called self-regulation skills [26] such as coping potential and reappraising the meaning of a bad situation [27] (p. 95). This also may count as resilience capacity, having the skills to regulate oneself and the potential to deal with a bad situation. Thus, resilience capacity would at least comprise interaction seeking and self-regulation skills, particularly coping potential (Figure 1).

Following from the two factors that make up resilience capacity, the resilience process would be to actually engage in interaction with the environment and to indeed deal with hardship that is encountered. Particularly the ability of problem-focused coping ("Let's address this situation") seems to increase the level of resilience [11], whereas emotionfocused coping ("Let's not get frustrated") and neuroticism cause adverse effects [11]. It turned out that, with emotionfocused coping, people perhaps expected less from life but this realization in itself made them feel lonelier [5]. 


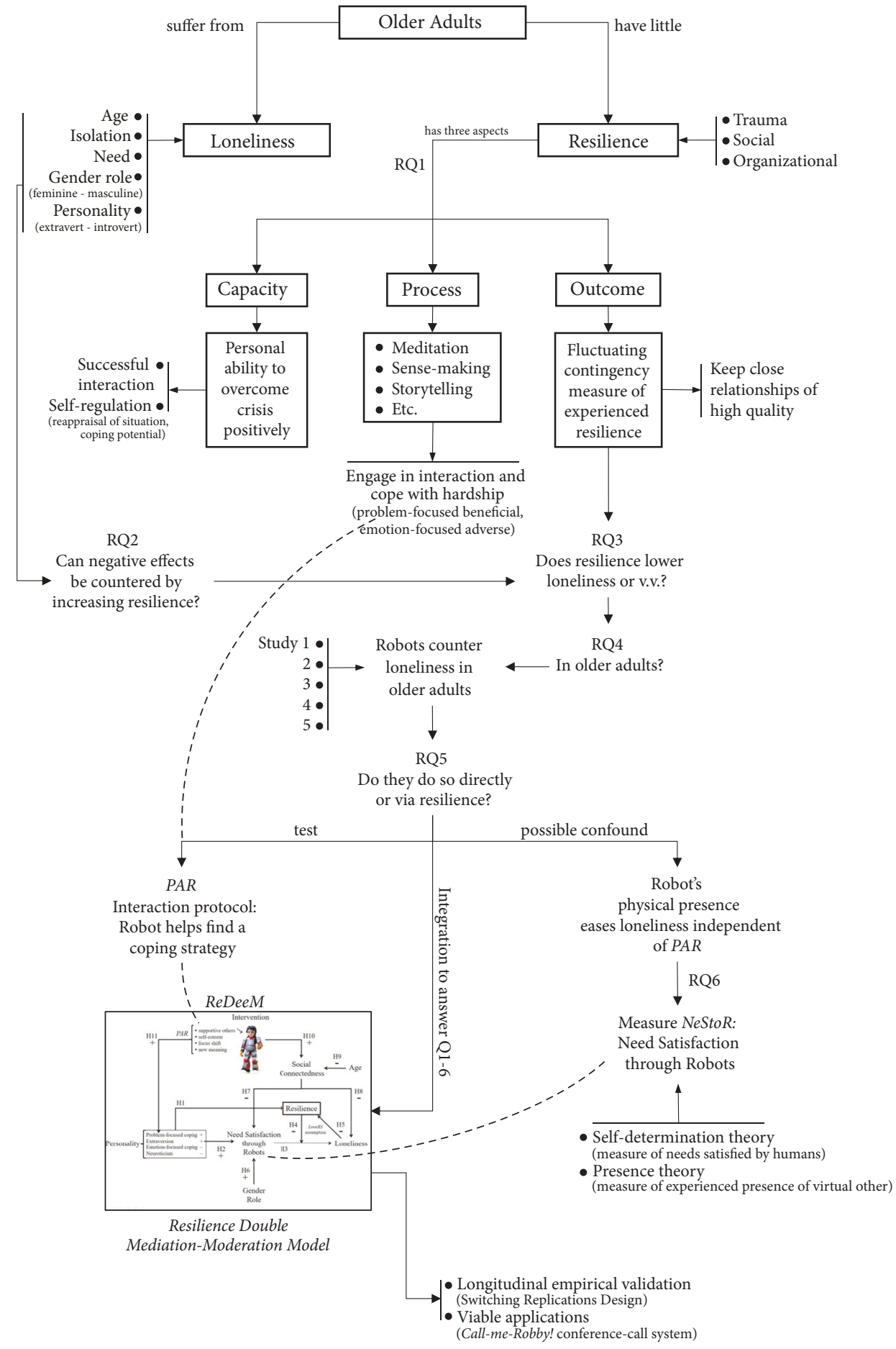

FIGURE 1: Conceptual overview of robot-supported resilience to lessen loneliness.

The outcome of the resilience process probably coincides with the environmental factor the literature mentions as being conducive to high levels of resilience: to keep close relationships with others [26]. Not having them is a crisis in itself. Close relationships offer emotional security [26] and instill hope [19]. They provide knowledge about what helps, what to avoid [26], and where to find resources [28]. A high level of resilience in itself moderates the negative effects that, for example, social neglect has on psychopathology such as depression and, indeed, loneliness [11].

\section{Loneliness}

Unlike personal resilience, studies on loneliness are bountiful. Loneliness diminishes physiological strength and increases health risks such as depression [3], dementia, and 
cardiovascular diseases. Loneliness predicts excess mortality [12], particularly in combination with depression [2]. Half the people of $80+$ experience loneliness [7]. The degradation of one's social environment, grieve, fewer visits from friends, and deteriorating health aggravate this chain of events [29]. Several factors seem to cause or intensify loneliness (Figure 1).

Being socially embedded is evolutionary most essential and social relationships should have at least a minimum level of quality [10]. When such criteria remain unsatisfied, loneliness emerges [10]. In self-determination theory [30], autonomy, competence, and relatedness are basic needs that motivate people to act. Satisfaction of these needs predicts personal wellbeing. Feeling lonely may be closely related to personality or may be a temporary circumstance [4] (p. 36). Not everyone has the same need for relationship. Introverts, for instance, may have fewer and less intense social connections but may not feel lonely. They connect in a less personal manner than extraverts do $[4,31]$ (p. 40).

Gender also may be of influence. Women on average live longer than men [32] (pp. 52-61) and tend to be more familyoriented. Women usually identify with 'feminine' qualities (whether derived from nature and/or nurture) such as wanting to care [33] (p. 297), although admittedly the feminine role of women is declining since the baby boom [34]. People with more feminine qualities (i.e., tender, nurturing, and caring) but who lack social contacts probably feel all the more lonely. More masculine, assertive, achievement and successoriented people (whether male or female) may feel less so.

All in all, loneliness has little to do with the number of people in one's environment [7] (p. 1) but rather with the quality of the contact [10]. In the absence of high-quality interaction, one may feel lonely while many come to visit.

This gives rise to the second research question (Figure 1: RQ2): can the influence of factors that increase the level of loneliness of older adults be pushed back by making people more resilient? Certain personalities seem to be more or less vulnerable to feelings of loneliness (e.g., extravertedintroverted). Loneliness increases with age and may be more severely felt when someone's orientation is towards the feminine. Social connectedness may ease the loneliness, provided that the quality of the contact is sufficiently high [10].

However, the literature also reports possible countereffects. It might be that in the face of adversity the level of feeling resilient is drained, indeed, by feeling lonely $[3,5]$. Put differently, increased levels of resilience may lessen feelings of loneliness. Or will high levels of loneliness be detrimental to feeling resilient? Or do both happen at the same time as a kind of self-enforcing vortex? The third research question, then, is (Figure 1: RQ3) how are levels of resilience and loneliness interrelated in terms of mediation, moderation, or direct effects? Are we dealing with an upward or downward spiral and, if so, how to interfere with its direction?

This brings us to the application side. To observe resilience in terms of successful interaction, self-regulation skills, and close relationships, or to study the way loneliness and resilience are intertwined, we need a test case. Many older adults have fewer and less profound interactions, skills, and relations. There may be a way out, however, if we can enhance their resilience to such standards that feelings of being lonely at long last are reduced. And so a fourth research question emerges (Figure 1: RQ4): can a general model of personal resilience be retrieved in an area such as loneliness in older adults? In what way does the application domain affect the model's structure?

\section{Robots (Boost Resilience to) Counter Loneliness}

In this section, I cite a number of studies that report effects of loneliness reduction in older adults, using social robots. In their literature review, Broekens, Heerink, and Rosendal [35] find that research methods are still immature and confounding variables lead to mixed results. However, these authors also state that quite a number of studies report less loneliness in older adults with a companion robot as assessed with loneliness measurement scales.

For roughly 15 years now, beneficial effects are reported using pet-like companion robots to counter depression and/or loneliness. Early longitudinal research by Wada et al. [36] found reduction of depression in older adults while using the PARO seal robot. Banks, Willoughby, and Banks [37] compared companionship of a real dog with that of the AIBO robot dog and did not find differences between the two: In both cases, loneliness was reduced as compare to a control group that had nothing. Bennett et al. also reported beneficial effects with respect to loneliness in older adults while employing the PARO machine [38]. Randomized controlled clinical trials with older adults showed that the company of a resident dog or the PARO robot reduced loneliness as compared to a control group who had neither [39]. Randomized controlled clinical trials in Hong Kong obtained better mood, more social interaction, and more communication in older adults with dementia after interaction with the PARO pet robot [40].

It seems, then, that robots can do what listening radio and watching TV cannot: robots can intervene with the older user in an interactive manner. In that respect, Dautenhahn, Campbell, and Syrdal [41] point out that it may not be the direct interaction alone that is helpful to reduce loneliness. It also may be the mediating role a robot fulfills, bringing people together and easing loneliness that way.

Robots apparently can counter loneliness through direct contact or by bringing people together. That from an applied viewpoint is good news. In view of the development of resilience theory, however, do robots counter loneliness directly or do they do so by enhancing resilience first, which in turn reduces loneliness? This would be my research question RQ5 (Figure 1). Or in view of RQ3 (Figure 1), does the reduction of loneliness actually increase resiliency, not the other way round?

In looking back at what we found so far robots may ease someone's loneliness but there are no studies stating that robots boost resilience, simply because that question was not asked before. Literature stated that a focus on problemfocused coping seems most beneficial to increase the level of resilience [11]. Thus, we should have a robot that offers its user the strategies to cope with setbacks in a problem-focused way (Figure 1). In other words, we need to develop a protocol 
for the robot to coach its user to resilience. That protocol, Pathways to Resilience or PAR is discussed in the next section.

However, the robots in the loneliness studies cited in this section did not have an intervention protocol or did not perform any deliberate actions to counter loneliness. They were just there. In other words, the robot's physical presence may ease feelings of loneliness without a resilience intervention needing to take place. This would count as a possible confounding part of any resilience intervention by a robot (Figure 1). Yet, with that protocol in place, we can test the magnitude of the effect of direct intervention on the level of resilience (Figure 1). Without the protocol, we can control the mere physical presence of the robot and to what extent a robot intervention with coping strategies installed has added value.

\section{Pathways to Resilience (PAR)}

If the robot is to interfere with the habits of a lonely person, it should have coping strategies ready when the user so requires. Therefore, we need a robot that is capable of stimulating problem-focused coping [11]. For a robot to execute such behaviors, it should run a so-called coping protocol by which it encourages resilience and through that eases loneliness.

Next, I will explore the first steps towards such protocol. The robot may use that to coach the user in dealing positively with life's challenges. This protocol is called Pathways to Resilience (PAR).

$P A R$ describes the process of resilience. To build a formal model of coping strategies, I start from Parsons et al. [42], who formulated an adaptive process of resilience as people deal with stress and adversity. Their 'mapping system' relates current situations to perceived needs and goals and checks what information is needed for response. If the desired result is not met, other means are selected to get a more adaptive outcome, taking earlier experiences into account. Executive strategies can be altered based on experience to adapt to an adverse situation.

Figure 2 illustrates that there is not just one road to resilience. It compiles a number of pathways, including strategies to help a person face life's setbacks. It might just be that these strategies when applied properly may constitute the high-quality social interaction that make people feel connected [7] (p. 1).

First, Figure 2 assumes that resilience may be a perception of one's individual approach to a situation; it may also be the collective perception of a group. Second, that perception may be internal (person or group looking at herself) or it may be external (person looking at another person). Those perspectives may differ. Someone may think s/he is fine although the doctor has diagnosed a serious illness. The first thing a social robot should ask with respect to resilience is whether people experience a setback at all.

Adverse situations could be the transition to a care institute. They could be environmental barriers, unsafe neighborhoods, migration patterns, inaccessible housing, and inadequate resources for socializing, which are all mentioned in response to feeling lonely [16]. People may be insecurely attached [43] (p. 222) or may be depressed, feeling lonely
[44]. However, if people do not experience this as a crisis situation (Figure 2: (1)), they are 'resilient' for the purpose of this model because in their view nothing is the matter, although an external observer may judge otherwise.

If someone does experience a setback (Figure 2: (2)), the question is what s/he will do (the 'strategies') to deal with the hardship. As coping strategy, the robot checks on supportive others ("Who always understood you well?" "Where did you meet them?"), exceptional events ("When did things go well?"), shaping possibilities ("What would you want?" "What do you like to do?"), and self-esteem ("What are your good qualities?" "Who speaks highly of you?" "When do you know things go well in your life?"). Users consciously think about these matters, shifting focus from problems to abilities, a reframing and reappraisal technique in family practice [27] (p. 95). Your daughter is not nosy, she is caring [27]. Deficits are relabeled as strengths, providing a new meaning to a bad situation, opening possibilities to shape a new context.

What someone prefers may be a matter of psychology, economic pressure, acculturation, or religion but the bottomline is the question whether it helped. Did employment of the strategy improve the situation? If so, that person finds him/herself 'resilient', although again, someone else may think differently. The loop continues because, in the new situation, new setbacks may happen that need to be dealt with.

If the person feels s/he did not improve (3), there will be no or less resilience felt. After all, all effort was for nothing. The question the social robot then poses is whether one would want to try another strategy if the previous one did not work. For some people this is impossible. They know but one way to respond and merely increase the effort in trying the same approach time and again (4). However, the situation may change through external factors so that in spite of repeating the same behavior the hardship passes by anyway (5).

If applying a new strategy to the old situation does work and the setback is overcome ("Setback? No."), the level of resilience rises. If not, the assessment of the adverse situation starts all over again, making a choice from the strategies the robot offers to change the situation.

An example to follow for implementation of $P A R$ is the FearNot! system for virtual storytelling that helps schoolchildren deal with schoolyard bullies [45]. In an adaptation of the FearNot! system, the robot will be capable of appraising the relevance of an occurrence, can independently choose an action, and ask the user what his/her related emotions are. If an event happens (e.g., the user is turned down on a date), the robot may query the user for goals that are achievable within the new situation (e.g., sing the blues) [38]. In view of the new goal, the robot selects an action. For instance, it approaches the user with a compliment or avoids a sensitive topic. It may also start a coping strategy (e.g., asking about supportive others: "Who are people who always understood you well?"). It also may attempt emotion regulation of the user, suggesting a reappraisal of the importance of the original goal so to moderate the user's stress levels ("He wasn't that nice anyway"). Next goes an example of a check by the robot after the coping intervention (Figure 2: PAR at point (3)), which I modeled after [46] 


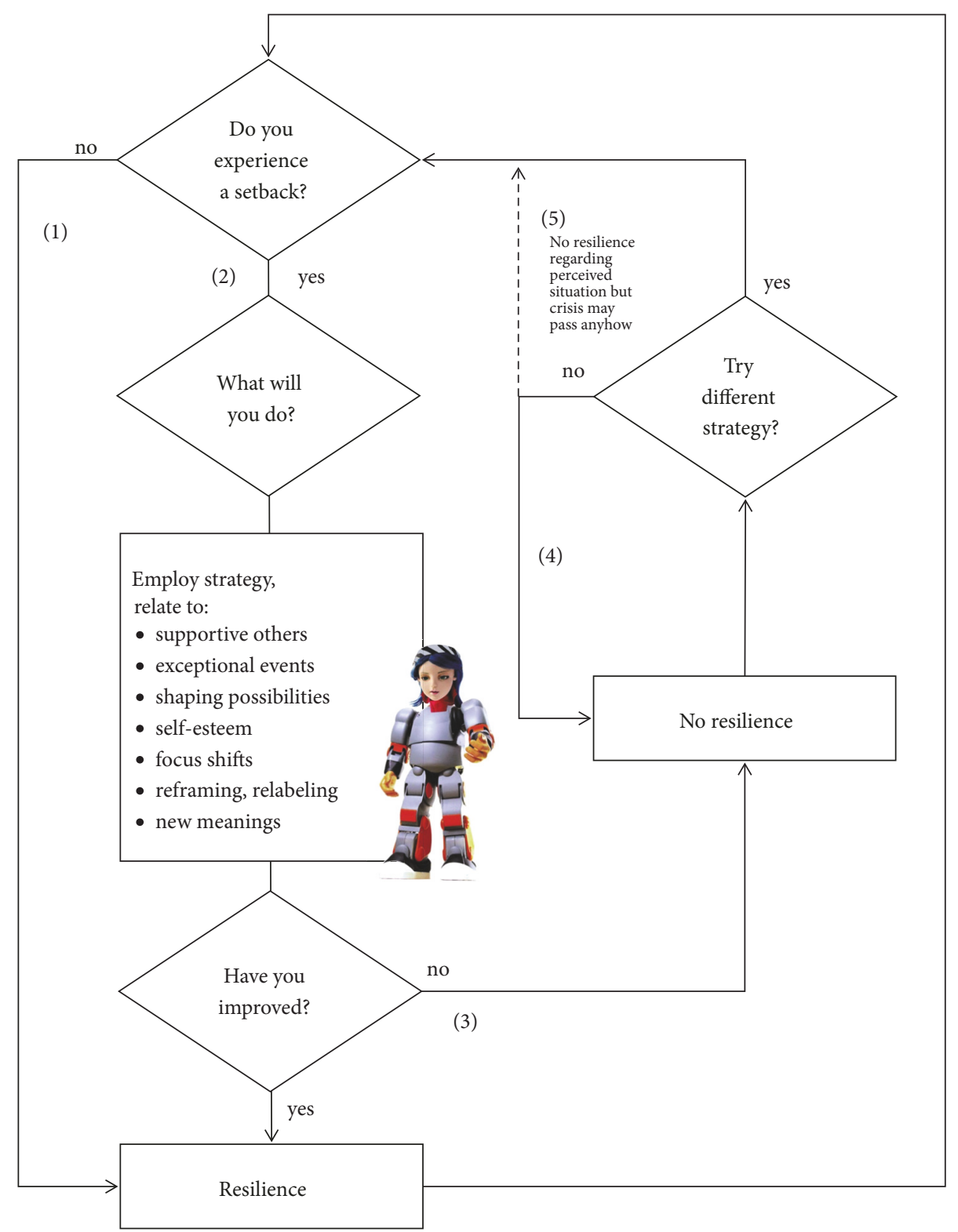

Figure 2: Pathways to Resilience (PAR). Robot may help find a strategy.

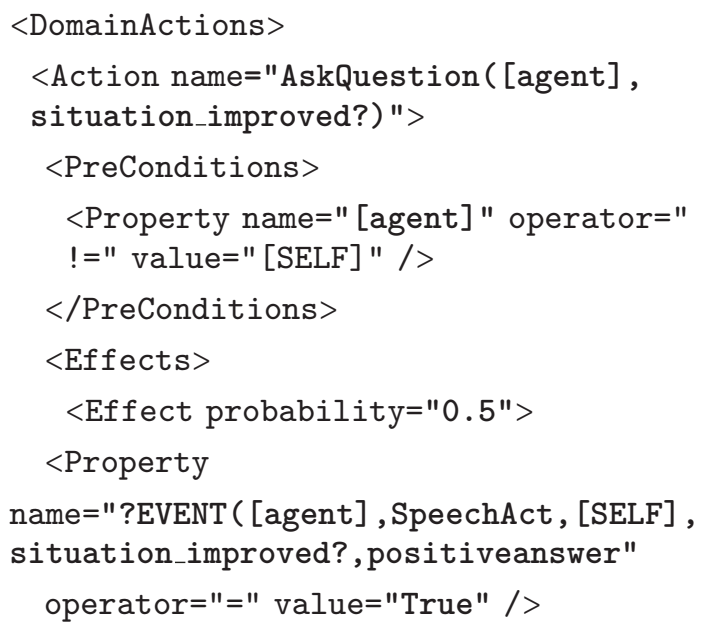

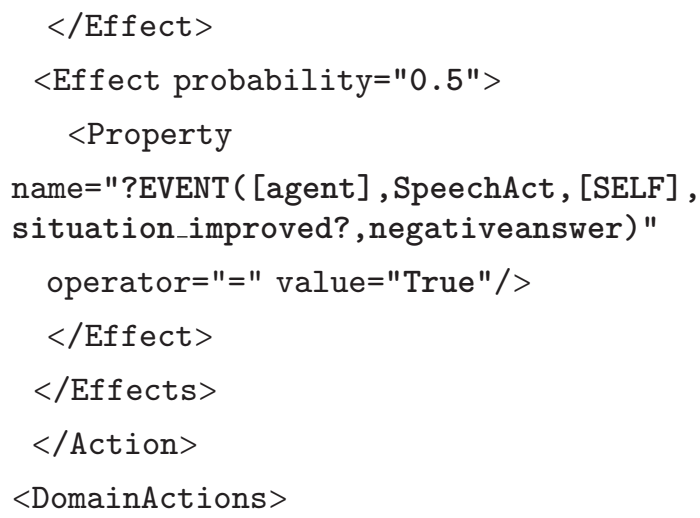

After a negative response by the user (Figure 2: Have you improved? No), the robot may select an empathic action of 
positive approach (p_app) to hug the user. The procedure again is modeled after [46]

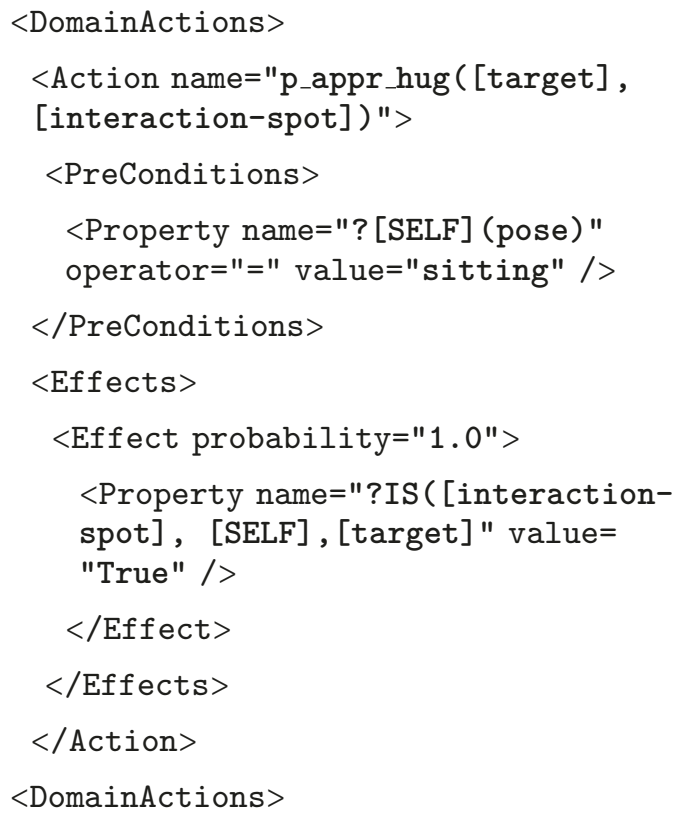

Automation of the coping protocol is convenient to systematically test effects of coping on resilience, which demands exact replication of the treatment for each participant. Exact replication is something a human confederate cannot deliver but a robot can. Additionally, the physical presence of the machine, its interaction possibilities, and human-like performance make a robot more suited as device to induce the treatment than, for instance, radio, TV, or Facebook. On the applied side, the repeatedly stated shortage of hands [1] in eldercare requires that a robot can support people independently $[8,47]$, for which some form of automation is indispensable.

\section{Need Satisfaction through Robots (NeStoR)}

Quite unexpectedly, the introduction of a social robot into resilience and loneliness studies also introduces a contamination problem. As mentioned in the section on loneliness and illustrated by Figure 1, loneliness transpires from the unfulfilled need for relationship with another human being $[30,48]$. However, studies show that robots already ease loneliness through their physical presence $[47,49]$. Thus, the need for relationship can be (quite surprisingly) compensated by a nonhuman, synthetic other [50]. Scientifically, it is germane to find out how much added value the robot's stimulation of coping behavior provides above and beyond the robot's physical presence. This introduces the sixth research question (Figure 1: RQ6): how does a robot make up for the need for human relationship and can we measure it?

Measures of need for relationship refer to humans, not to robots. To answer RQ6, measures of need for relationship should be adapted to whether 'the robot fulfills that need' (need satisfaction through robots). This also opens the possibility to study whether a robot (partly) can make up for a degraded social network.
In view of RQ6, it would be wise to construct a new set of questionnaire items based on the need for human relationship [48] (p. 53) but combined with measures to evaluate the social presence and appeal of synthetic characters (e.g., virtual agents, robots) [49]. The new scale that measures need satisfaction through robots is called NeStoR.

The 'need' part of NeStoR is based on self-determination theory: people have a basic need for relatedness; feeling accepted by and important to others, to feel cared for, and to care for them [48] (p. 53). In line with RQ6, indicative items will address being cared for, being acknowledged, and feeling a sense of belonging; counterindicative items should address feeling isolated, misunderstood, instrumentally used, and unconnected.

To measure NeStoR, an adaptation could be made of the Relatedness scale from the Basic-Need-Satisfaction(General) questionnaire [30]. For example, "I really like the people I interact with" and "I consider the people I regularly interact with to be my friends" become "I really like the robot I interacted with" and "I consider the robot to be my friend," respectively.

These human-oriented items are then connected to social presence of synthetic characters, the experience of "being with another" [49], as if someone else is there although not in person. This is the 'robot' part of NeStoR. The companion may not even be a person as long as some social actor is physically present in the room and responds in a human-like manner. This enhances the effect of having real company and that a positive interpersonal and emotional connection between communicators is established [51].

NeStoR, then, makes use of this sense of coexistence, of human realism, probing the degree of awareness of the artificiality of the social interaction with the robot, or as counterindication, the unawareness of the social mediation [52]. For example, "I felt like I was in the presence of another person in the virtual environment" [52] becomes "With the robot, I felt like I was in the presence of another person."

It may be that the need NeStoR assesses is different for different people. Perhaps feminine and introverted people are satisfied by robot company but masculine and extraverted people are not. In other words, we can cross-tabulate the factors that cause of aggravate loneliness and arrive at the predictions for NeStoR in Figure 3. The plot shows the expectation that personality (extravert-introvert), gender role (masculine-feminine), social connectedness (intactdegraded), and age (young-old) have a (possibly curvilinear) relationship with NeStoR. Figure 3 indicates to what extent a surrogate can make up for the wish to have real people around that offer the right form of social connectedness.

In Figure 3, NeStoR increases when people are more introverted and are more feminine and when the quality of their social connections is degraded, which happens when they grow older. In Figure 3, the linear-relation hypothesis is summative in that each factor (level) adds to NeStoR in equal measures. The hypothesis on curvilinearity assumes synergistic interactions, where the combination of factors is stronger than the sum of their effects. Linearity is the simplest assumption; curvilinearity may be more realistic [53]. 


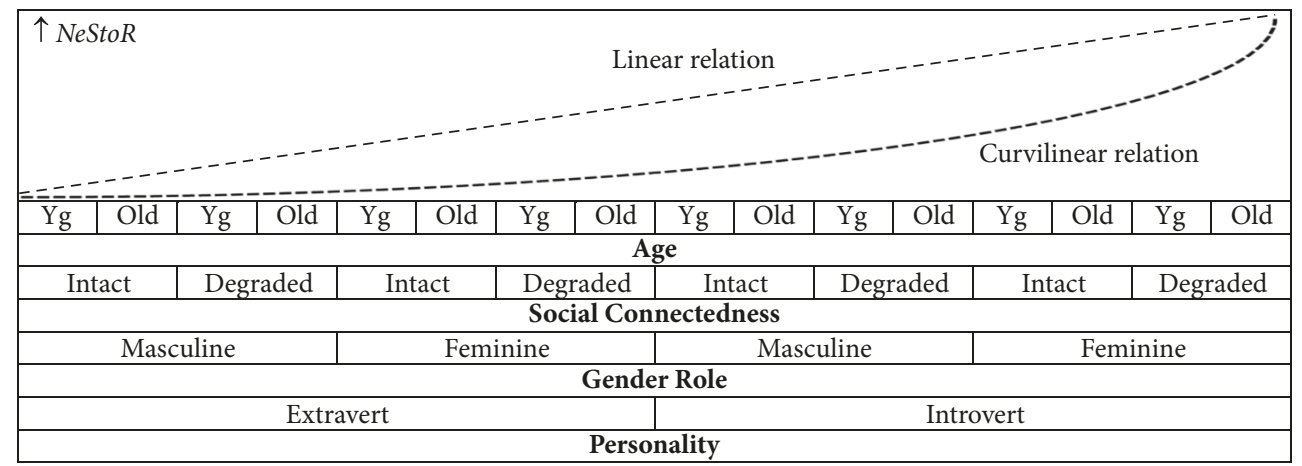

Figure 3: NeStoR as a function of socioindividual conditions.

\section{Resilience Double Mediation Moderation Model}

The overall objective of the current paper was to establish a new theory of personal resilience integrated with the factors that affect loneliness. That theory should inform humanhumanoid robot interaction (HHRI) to explain loneliness reduction and the build-up of resilience in older adults through social robots.

To answer RQ1-6 (Figure 1), I formulated a new model of personal resilience: ReDeeM (Resilience Double Mediation Moderation Model). With ReDeeM (Figure 4), I try to do justice to the insights and findings from the research literature regarding personal resilience while combining that body of knowledge with the one on loneliness. Whereas $P A R$ described the process of resilience, $R e D e e M$ describes the level of resilience as an outcome and a moderator of other factors. It includes the $P A R$ protocol by means of robot intervention. Next I formulate a number of hypotheses $(\mathrm{H})$ that are represented by relations (arrows) in ReDeeM (Figure 4).

Backbone of ReDeeM is the empirically established relation between personality and resilience (H1), particularly the ability of problem-focused coping [11]. By contrast, emotionfocused coping and neuroticism were found to have an adverse effect on resilience [11].

Personality also has another effect $(\mathrm{H} 2)$ : literature states that more extraverted people have stronger needs for relationship than introverts [4] (p. 40). When such need is unfulfilled, loneliness increases $(\mathrm{H} 3)$. The route from $\mathrm{H} 2$ to $\mathrm{H} 3$ in Figure 4 shows the first mediation: the factor need satisfaction through robots (NeStoR) brokers the relationship between personality and loneliness.

However, literature shows that high levels of resilience moderate the negative impact of emotional neglect on psychiatric symptoms such as depression [11]. Likewise, resilience may moderate the relation between need satisfaction and loneliness (H4).

Yet, I also suspect that loneliness may backfire (cf. [5]), causing resilience to drop (H5). I dubbed this constellation the Loneliness-Resilience Spiral or LoneRS assumption (see RQ3), describing an upward or downward spiral that reinforces itself. If so, then the LoneRS assumption represents the second mediation in the model with loneliness in between need satisfaction and resilience.
Social factors such as gender role may increase the need (to care for, being relevant to others) (H6). How well do people fit into the traditional gender stereotypes (acculturation vs. personal inclination)?

Additionally, the quality of social connectedness satisfies need satisfaction (H7) and may be a direct cause of not feeling lonely any more (H8). Care should be taken to focus on the quality of relationship (i.e., emotional closeness, self-disclosure) rather than the frequency of contact or the number of people surrounding the older adults.

With age, social connectedness declines (H9) for which an intervention through social robots may compensate (H10). There are two sides to the robot intervention. Literature shows that its physical presence provides a sense of companionship [47, 49]. Second is the behavioral change the robot will attempt, offering strategies that encourage problemfocused coping in older adults (H11), which supposedly was the prime source of improving resilience [11]. In sum, I offer the hypotheses of $\operatorname{ReDeeM}$, where $(+)$ indicates a positive correlation and (-) a negative correlation:

(H1) Personality (i.e., problem-focused coping) enhances resilience $(+)$

(H2) The more extraverted, the higher the need (+)

(H3) The more the need is satisfied, the less lonely one feels $(-)$

(H4) Resilience moderates the adverse effects of unfulfilled need on loneliness (cf. [11]) (-)

(H5) Reduction of loneliness leads to increased resilience $(-)$

(H6) The more femininity, the higher the need (+)

(H7) The less connected one feels, the greater the need (-)

(H8) The less connected one feels, the lonelier one feels (-)

(H9) With age, social connectedness deteriorates (-)

(H10) Physical presence of the robot enhances connectedness (+)

(H11) Robot's coping strategies enhance user's problemfocused coping $(+)$

(H12) The combined effect of social-individual factors on need satisfaction (through robots) is either linear (H12a) or curvilinear (H12b) (Figure 3) 


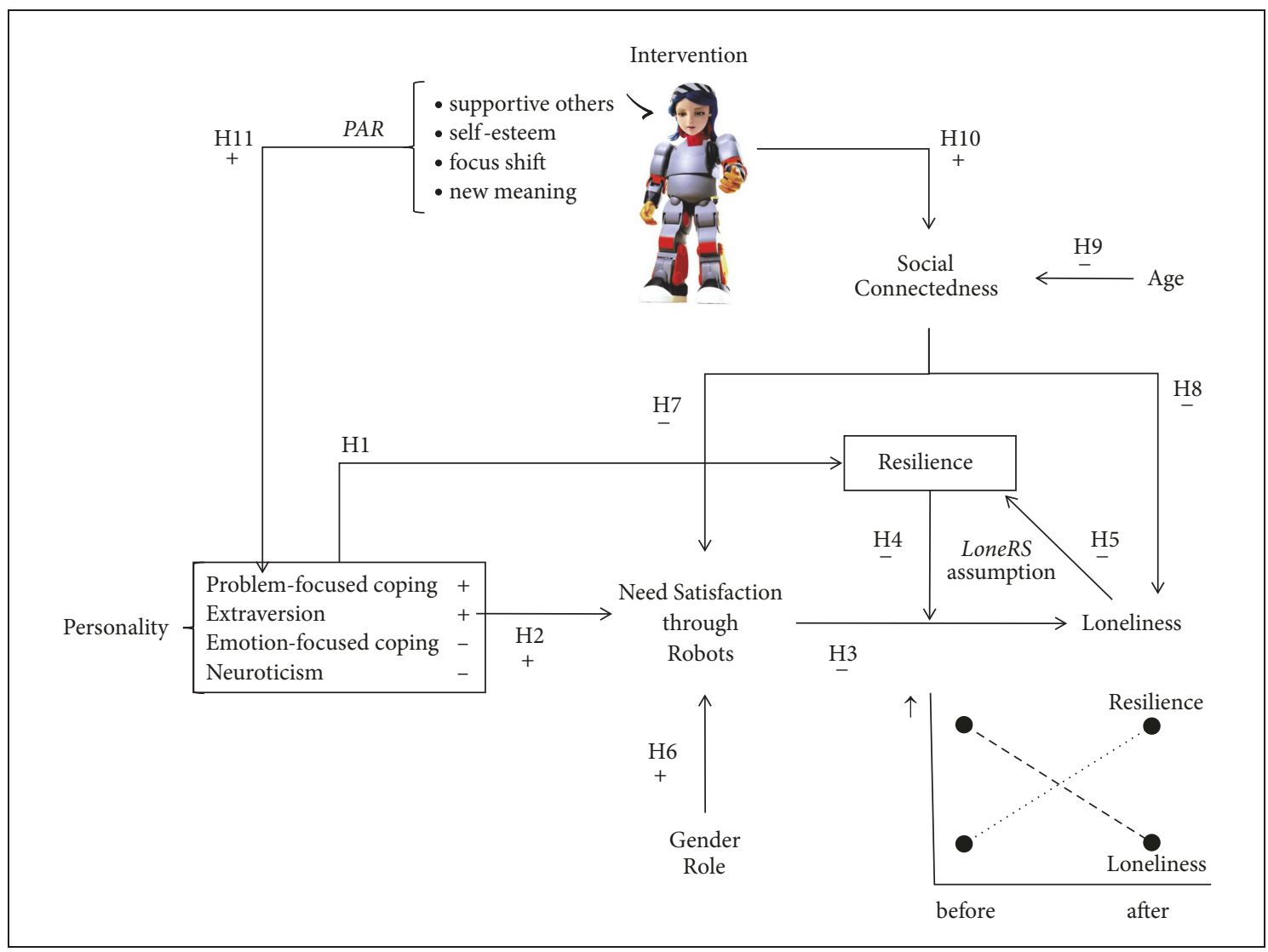

FIgUre 4: Resilience Double Mediation Moderation Model (ReDeeM) before/after robot intervention.

\section{Materials and Methods}

In this section, I offer a method to test the effectiveness of robot intervention in a longitudinal setting (Figure 1). Readers should be aware, however, that no actual data will be presented because longitudinal studies take years of sampling. This section is more of a discussion piece of how to approach issues of methodology before doing the actual work. With hypotheses derived from the novel theoretical framework of resilience, the method should follow a switching-replications design in which video footage of older adults in interaction with robots are rated by 'blind' observers for resilience, loneliness, etc. at three moments in time.

8.1. Participants. Pew Research Center investigated over 7000 US seniors for communication technology use and found a diverse picture [54]. Currently, roughly $25-50 \%$ of the seniors of 70+ own a cellphone and smartphone, are on the Internet, or use broadband services. About 33\% own a tablet or computer and are active on social media. Yet, a larger number (50-75\%) have a distant relationship with technology, more so the older, less educated, and financially vulnerable they are. Older adults report not to be skillful; they face physical barriers (e.g., fingers, eyes) and rely on others to set up technology and show its usage. However, those who use communication technology state that it enriches their life and positively impacts society [54].

Given this positive attitude but negative aptitude, technology that supports older adults should be telephony based, the best known option to this group. Computers and tablets may be functionally appropriate to look up information or to chat [55]; however, operating the interface may be a barrier. One should be able to talk to it, not type, swipe, scroll, click, or browse it. Moreover, the machine should explain itself, literally.

With age, friends and family become fewer and the barrier to contact a stranger becomes higher. Therefore, the machine should make connections independently, leaving the decision to engage with the older adult. Therefore, I propose to let a robot make the call, facilitating social connectedness, looking up information, and providing active support through PAR. With the phone on the hook, the machine has physical humanoid presence, serving as a social surrogate when no one is around.

Because females are the larger group and most have played a feminine role during their lifetime, I focus on $80+$ females with an introverted personality, an impoverished social network, and a diagnosis of pathological loneliness. Because introverts connect in a less intimate way [4] (p. 40) and tend to connect more indirectly through technology [31], robot companionship may work well for them. 
8.2. Design. With respect to experimental design, I want to discuss the combination of qualitative and quantitative data and their respective means of sampling. Older adults find laboratory experiments and filling out questionnaires rather challenging [56, 57]. However, observational casestudies render data of low reliability and generalizability [58]. Therefore, I propose a mixed method in which qualitative observations are quantized by 'blind' observers (not family or care professionals) in an experimental setting. As Happ puts it: “...there is little standardization of mixed methods research practices and relatively little guidance for data combination or integration. This is an area that provides opportunities for methodological creativity..." [56].

In mixing qualitative and quantitative methods, four levels are discerned [58]: (1) separate data collection and analysis, (2) results of one method inform the other, (3) comparison of separate results, and (4) merging data sets through data transformation followed by additional analyses. Regarding 2, ample examples exist that in geriatric studies quantitative data are used in a qualitative follow-up to evaluate the ecological validity of the numerical results ("qualitizing the data") [56]. I do things the other way around (4) by "quantizing" the qualitative data.

In Hoorn [59], I proposed videotaping older adults in interaction with the robot in a switching-replications design. Next, I elaborate upon the idea so that some parts show some overlap with [59]. I propose using the video recordings as a stimulus set in a structured-questionnaire study in which a large sample of 'blind, not family-biased, observers assess the behaviors of the interactants. The results are open to robust statistical techniques (e.g., Analysis of Variance, Structural

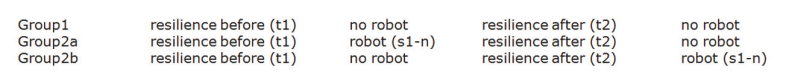

Because Groupl does not see any problems, predictions for the outcome variables are absent except that perhaps the level of resilience remains about the same at all time points $(t 1 \approx t 2 \approx t 3)$. The difference with Group $2 a$ and $2 b$ is not so much a hypothesis as it is a research question. However, the data sampled in Groupl (together with the data of Group2a and $2 b$ ) certainly can be used to evaluate the relations (H1H12) predicted by ReDeeM (Figure 4). With Group2a, the long-term effects are tested of robot intervention (employing strategy 1-n). With Group2b, we test whether having no robot companionship at $\mathrm{t} 1$ and $\mathrm{t} 2$ worsens loneliness and resilience and whether that improves after receiving a robot at $\mathrm{t} 3$.

Whether differences between Groups (2a vs. 2b) are significant is inconsequential. It may be that robot intervention in total renders the same effects in Group 2a and $2 \mathrm{~b}$. However, the statistical interaction between Groups (2a vs. $2 \mathrm{~b}$ ) and Times (t1 vs. $\mathrm{t} 2 \mathrm{vs.} \mathrm{t} 3$ ) of measuring resilience is critical. Hoorn [59] predicted the following systematic: a lift-off-level-out effect (resilience at $\mathrm{t} 1<\mathrm{t} 2 \approx \mathrm{t} 3$ ) would sustain hypothesis $\mathrm{H} 11$ that in Group2a robots inspire resilience in
Equation). This is different from common approaches where three raters categorize observations according to a protocol (cf. Atlas Ti), which is too dependent on interview style and grouping of concepts [58]. My approach leaves older adults in their homes and yet renders reliable measurements (i.e., scale analysis), allowing for full model fit as well as the analysis of longitudinal effects. It is literally so that “... no longitudinal studies found to date have tracked changes in resilience over time among older adults" [60] but my approach may make this happen.

Happ has a point when she observes that it is difficult to quantize qualitative results when probabilities of occurrence of a phenomenon (e.g., 'resilient behavior') are unequally distributed over the sample [56]. Therefore, we should not rely on just one session with one older adult but make a large stimulus set of comparable replications that moreover is judged by more than the standard three raters, making the set-up a field experiment with laboratory characteristics with all the possibilities to evaluate the reliability of the measurements: "The next generation of mixed methods research in gerontology is challenged to combine approaches in ways that lead to responsible data integration (Level 4)" [56] and that is what I propose.

Senior Groupl $(n=32)$, without robots, is the control group and Group2 the treatment group, interacting with a particular robot arrangement (see Apparatus/Materials). Group2 $(n=64)$ will be split in two $(n=32$ each). They are divided over a switching-replications design. This is a beforeafter repeated-measures design with and without robot intervention (strategy sl-n). The most important dependent measures are resilience and loneliness at time point t1-3 but all $R e D e e M$ variables can be measured and analyzed this way:

older adults. The effect of $\mathrm{t} 2 \approx \mathrm{t} 3$ would indicate that after initial increase by robot treatment, people in Group2a found a way to maintain higher levels of resilience independently. A lift-off-boost-up effect also would corroborate H11, observing a steady increase in resilience at $\mathrm{t} 1<\mathrm{t} 2<\mathrm{t} 3$. The second increase effect between $\mathrm{t} 2$ and $\mathrm{t} 3$ would point out that, in Group2a, the robot inspired and taught the skills to accelerate people's own resilience without the robot necessarily being present afterwards. This would be the most beneficial effect of the robot intervention. H11 is supported as well, if $\mathrm{t} 1 \approx \mathrm{t} 2<\mathrm{t} 3$, an incubate-accelerate effect. This is the least beneficial effect because robot support may be continuously needed although that is a worthwhile finding still. All other outcomes may be considered a refutation of H11 [59].

8.3. Apparatus/Materials. As said, 'loneliness can have maladaptive effects on behavior' [7] (p. 2). When older adults notice that social networking, video calls, and smartphones reduce loneliness, they appreciate new technologies [15]. Probably, this will be even stronger when functions are 


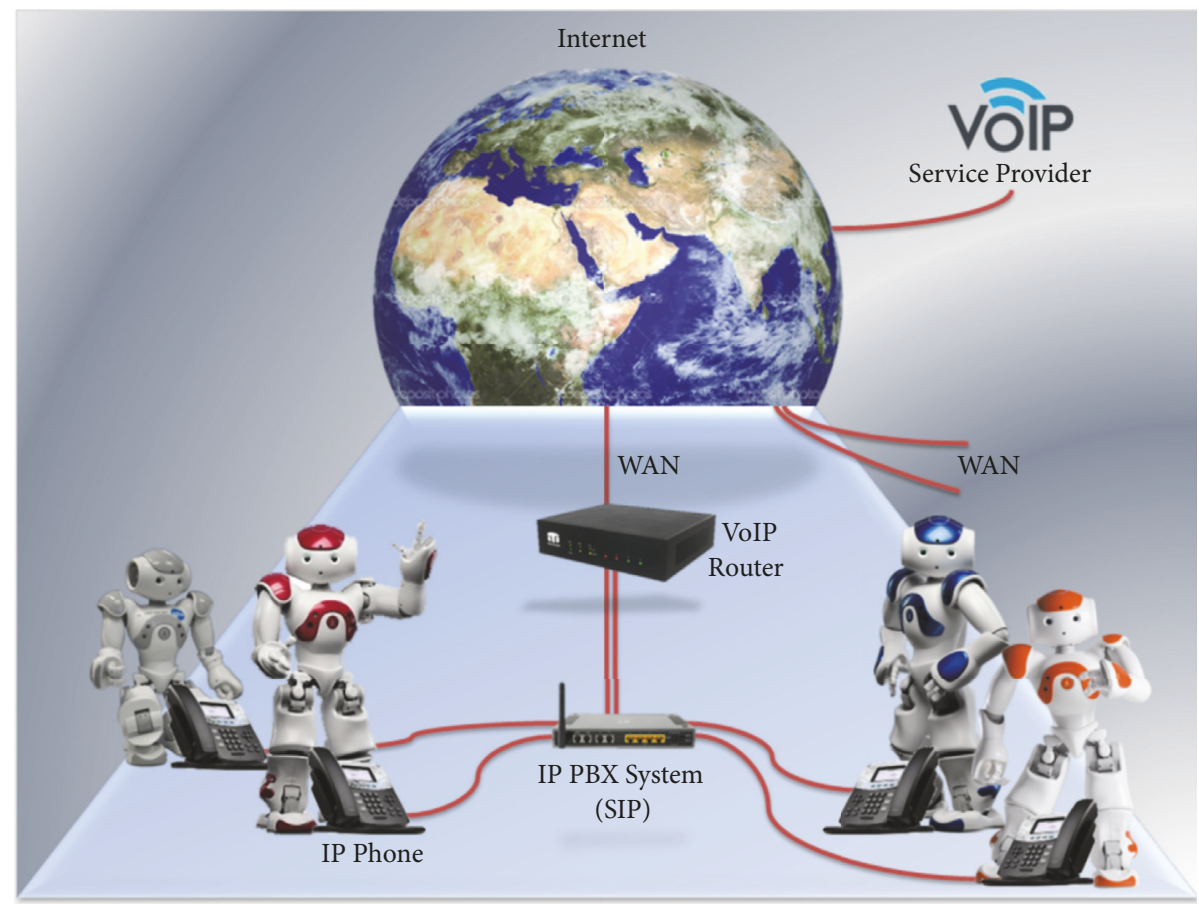

FIGURE 5: Configuration of the Call-Me-Robby! conference-call system.

articulated by a physical presence, with eyes and voice [61, 62]. Digital communication in home care is for contact, work meetings, and arranging care [63]. Social robots could help start the contact network, which later may evolve into a meeting or arrangement network.

Shy users may not likely pick up the phone and make a call. Therefore, I am working on a conference-call system (Figure 1) with (initially) four robots, conversing with each other over the phone while four older users overhear the conversation, anonymously at first, but they can identify themselves at any moment. The conversation takes place in speakerphone mode.

Call-me-Robby! shifts the burden of feeling as a burden from the user to the robot, making the user's problem the robot's. The robot wants to make a call to a fellow robot ("Alice wants to chat with Zora") and the users listen in while the robots discuss user problems as if they were their own. Users are free to break in any time and add to the conversation, also with perfect strangers that, as a conversation piece, have a robot linked in the network as well. Note that seniors can stay at home if they want: the contact is telephonic, not necessarily in person.

Because the target group is used to conventional telephones, Call-me-Robby! will be composed of a set of IP phones (Figure 5), which look just like regular phones, except that IP phones use an Ethernet connector (type RJ-45) to a SIP Server or IP PBX System. In using Voice over Internet Protocol (VoIP), the PBX communicates with a VoIP Router that has a WAN connection to the Internet where the VoIP Service Provider handles the calls. This set-up serves as a standalone conferencing service for experimental purposes and can be connected later to other groups, using multiple WAN connections.

Each robot (e.g., $A^{\prime}$ ) speaks on behalf of its user (e.g., (2) to express the problem ("We do not want to leave our home") but serves as sparring partner for the other user (e.g., B), running the $P A R$ process. Reversely, the other robot (e.g., 웡 B') represents the second user ( B), serving as sparring partner of the first ( A). As soon as the users take over the conversation, the robots keep quiet or take up where they left when human conversation stops.

As to the design of the robot's personality, introverts may not like an interruption by a robot, whereas extraverts appreciate more facial cues and expressiveness. Similar personalities between robot and user attract [64] but complementarity attracts as well [65]. A pilot study should determine what the robot's character for introverted older women should be.

Apart from being the matchmaker, the robot plays another, rather special, social role. Older adults are short of confidant friends [66] but also seem more satisfied with the friends they still have [67]. A friend is someone you can trust, who will not judge you, who supports you, who has the patience to listen, and who does not claim social space. A social robot can do this by and of itself [47]. A robot is not disturbed by thought or emotion and remains in a constant state of no-mindedness ('Zen'). The robot should play the role of a trusted confidante, advising how to overcome adversity, asking open questions ("What is the problem? What will you do? Is it better now?"), and inviting self-disclosure.

If the user experiences a setback (e.g., "I can't live at home anymore. I have to leave"), the robot runs through its 
coping strategies (Figure 2). If one strategy fails and the user is willing, the robot tries a next. To guide the robot's behaviors it could follow Acceptance and Commitment Therapy (ACT) [68]. The robot promotes mental flexibility by letting users notice what goes on in their minds, accepting undesired thoughts and feelings as much as the desired ones. The robot will follow emotion regulation behaviors [69] and attempts problem-focused coping and adaptive problem solving [44]: it will mention supportive others and exceptional events, shaping possibilities and self-esteem and shifting focus from problem to ability and to the reappraisal of the situation [27] (p. 95).

The robot checks the loop "Did you improve?" and, if "No," asks to try a different strategy (Figure 2: (3)). Irrespective of the outcome (i.e., more resilience or not), users engage in making contact with the help of the robot, either to complain about their situation, the robot, or to discuss their new perspective. At least there is content to converse about and a reason to call and become socially connected, which strengthens resilience anyhow.

8.4. Procedure. $P A R$ assumes a group that experiences no setbacks, which may be regarded as 'resilient.' This is Group1 $(n=32)$, who need no robot interference (Figure 2, path (1)). The second group (path (2)) does perceive an adverse situation and will be exposed to robot intervention. This Group2 is doubled $(n=64)$ for experimental purposes (see Design). Therefore, the target is to have $N=96$ seniors partake in the experiment.

As suggested in Hoorn [59], three moments of measuring ReDeeM (Figure 4) are planned. One hour of video surveillance observes participants in their homes. Afterwards, the footage of that one hour preperiod is cut back to about 15 minutes. After the preperiod, a trained interviewer asks open questions for about 10 minutes: "How do you feel? Is life good to you?" This session is recorded as well but the interviewer is not on camera.

The preperiod is spent without robot intervention in Group1 whereas Group2 does receive robot treatment. In their preperiod, Group2 trains on handling the robot for the first 15 minutes. Then the robot runs PAR strategies for about 30 minutes after which they spend 15 minutes using the Call-me-Robby! application. With four seniors in the loop and $n=64$ in Group2, sixteen independent Callme-Robby! sessions will take place. Because we build our own portable IP Phone configuration, participants can stay at home and do not need to travel to the laboratory. As said, each senior is videotaped (requiring four cameras) while they are in the conference call and during the interview thereafter.

The shortened video footage of the period preceding the interview (the 'preperiod') and the interview itself (together about 25 minutes) are the stimulus materials that are viewed by observers unrelated to the senior to assess the state of mind of the senior, using the ReDeeM structured questionnaire (see Measurements). Both clips and items are presented in pseudo-random order.

For $N=96$, we plan for $150+$ observers, viewing 96 clips of 25 minutes each, after which observers fill out the
ReDeeM questionnaire ( $\pm 10 \mathrm{~min}$.). This results in 40 hours of observation time plus 16 hours of assessment, making 56 hours of work spent by one observer after one wave. Because measurement is repeated twice, $3 \times 56=168$ hours of work per observer is spent, making a grand total of 25,200 person hours.

8.5. Measurements. The online questionnaire that measures ReDeeM will consist of 7 measurement scales (6 items each, including 3 counter-indications), age, education, and other demographics. The questionnaire will be structured according to [70] and filled out in Qualtrics by the observers to score the videotaped behaviors in the preperiod and during the interview. For Groupl, the preperiod is filled with everyday activities; for Group2, it is the robot conference call.

Personality is assessed with a shortened version of the revised Eysenck-Personality Questionnaire [71]. For problem-focused coping, I will look into the CopingInventory [72].

To measure NeStoR, I adapt the Relatedness scale from the Basic-Need-Satisfaction-(General) questionnaire [30]. For example, "I really like the people I interact with" and "I consider the people I regularly interact with to be my friends" become "She really likes the robot she interacts with" and "She considers the robot she interacts with to be her friend," respectively. This is in combination with items on social presence [49].

For the Social Connectedness scale, I draw on the Inclusion-of-Other-in-the-Self scale [73], SubjectiveCloseness-Index-and-Relationship-Closeness-Inventory [74], and Personal-Acquaintance-Measure [75]. I will focus on the quality of relationship (i.e., emotional closeness, self-disclosure) rather than frequency of contact or number of people surrounding the older adults.

For the assessment of loneliness, I will look into the Loneliness-Rating Scale [76], the De-Jong-Gierveld-Scalesfor-Emotional-and-Social Loneliness [77], the Differential Loneliness Scale [78], and the UCLA-Emotional-versusSocial Loneliness Scales [79].

To evaluate beliefs about Gender Roles, a subset of the Bem-Sex-Role-Inventory (BSRI) will stipulate the participants' gender role as more masculine or feminine, androgynous, or undifferentiated [34]. How well do the women fit into the traditional gender stereotypes (acculturation vs. personal inclination)?

For resilience as an outcome, a Resilience Scale will be distilled from the Connor-Davidson-Resilience Scale, the Resilience-Scale-for-Adults [80], and the Brief-ResilienceScale for resilience to anxiety and stress [80].

Control items probe for differences during the preperiod (older adult in intuitive mode) and during the interview itself (reflective mode) and for novelty as a covariate, which should diminish towards $\mathrm{t} 3$. After scale analysis, a mean index is calculated for each ReDeeM variable for that particular older adult in each wave (t1, t2, and $t 3)$. From the grand-mean averages, GLM Repeated Measures will estimate before-after effects of the robot intervention on resilience, etc. Multiple regressions (i.e., mediation moderation analyses [81] and Structural Equation) test the structure of ReDeeM. 


\section{Results and Discussion}

Note that the previous sections were meant to instigate a discussion on theory and methods in the study of resilience through robots. After testing is completed (in the future), conclusions may be drawn to design a health intervention by a social robot (Figure 1), which would be a novelty in health sciences. In particular, results of the tests of theory (ReDeeM, PAR), methods (NeStoR, switchingreplications), and technology (Call-me-Robby!) should be informative to the design of an HHRI resilience-loneliness intervention.

Systematic reviews of resilience interventions are scarce and mainly address youngsters [60]. However, factors enforcing resilience partly overlap with those countering loneliness: they include adaptive coping, optimism, hope, positive emotions, social support, community involvement, and autonomy [60], factors mentioned also in APA's Resilience Tool Kit. Most resilience interventions attempt to raise positive emotions through, for instance, education, therapy, group activities, screenings, and counseling [60] but none enhances resilience through technology, let alone social robots. Without much difficulty, robot technology can be personalized, a quality that is most wanted in resilience programs for older adults [60].

Systematic reviews of interventions for loneliness and isolation state that effectiveness can be hardly assessed because studies are not so well conducted [82]. I want to fix this problem by using switching replications. Effective intervention should be grounded in theory (ReDeeM, PAR), provide social activity in groups, activate older adults to partake [82], and emphasize adaptability, community development, and productive engagement [83], all of which are present in Callme-Robby!

There are but a few technology-assisted loneliness interventions, most of them involving computer training to communicate with relatives and friends and to search for information. Review papers state that research quality is weak, making the results inconclusive $[82,84]$. Telephonesupported interventions mainly concern help lines (cf. suicide) and decrease depression but not loneliness. Telephone circles among women or with informal caretakers that facilitated social support did not help against feelings of isolation, loneliness, or depression $[6,82,85]$.

Probably, those initiatives take care of the abstract information side of treatment but not of the physical presence of a social entity. This is why in application, Call-me-Robby! wishes to improve social skills, social support, social contact, and maladaptive social cognition, which is the most effective remedy against loneliness [86].

A limitation of the current approach is that PAR does not keep record of the development of the resilience process. It does not have a 'mental model', let alone a 'theory of mind' of its user. As a second challenge, $P A R$ also may have a model of the user's environment (cf. situation awareness) to assess possible crises. It is no trivial task to connect visual and linguistic input to such models and not solely rely on the assessment of the user.

\section{Conclusions}

In view of the concerns forwarded by Frennert and Östlund [9], this paper addressed the social role of humanoid robots as confidante and coach in older adult's lives. To develop an effective interaction paradigm in HHRI, the current paper also addressed a new HHRI research methodology for longitudinal study, which unobtrusively involves older adults in the development of their social robots. The paper also provides conceptual clarity in matters that concern older adults the most: loneliness reduction and the reinforcement of resilience. By using the robot as an intermediary in human-human contact, hopefully, I circumvented technological determinism by allowing the formation of a new social construction of humanoid robots [9].

From the explorations in human-humanoid robot interaction with regard to loneliness and resilience support, the following conclusions can be drawn (Figure 1). Older adults have little resilience due to trauma (e.g., broken hip), social setbacks (e.g., deceased husband), or organizational distress (e.g., changing rooms). They often suffer from loneliness as caused or aggravated by age, social isolation, unfulfilled needs, gender role, and personality. Resilience is a tripartite concept. Resilience encompasses capacity (successful interaction, self-regulation) to overcome hardship in a positive manner, a process to engage in interaction and deal with setbacks (i.e., problem-focused coping), and an outcome, which is to establish and maintain high-quality relationships.

Humanoid robots can support the problem-focused coping strategies, using the protocol Pathways to Resilience (PAR) as powered by procedures derived from the FearNot! affective computing system. One of the new research questions is whether humanoid robots address loneliness directly through physical presence or that they take the detour through increasing resilience. With the new measure need satisfaction through robots (NeStoR) that question can now be answered. This is because NeStoR's subscales rely on self-determination theory to probe the humanoid side of robots and on presence theory to probe their virtual side.

Everything becomes integrated into the brand new Resilience Double Mediation Moderation Model (ReDeeM), opening up the possibility of future empirical validation of HHRI in a systematic and hypothesis-driven way with respect to human loneliness reduction and resilience development through humanoid robots. To facilitate that research with fragile older adults at home, a novel, indirect, and observational method was devised, quantizing qualitative samples in a switching-replications design. Finally, as a new way of interaction, an HHRI matchmaking system was proposed that takes the burden of asking for contact from the human user and puts it on the robot, providing the user a safe environment to explore novel human-human relationships without showing or losing face.

Looking onward and into the future of human-humanoid robot interaction (HHRI), many interesting events may occur that will require further examination. In a broader vision than conventional HRI, how as human senders of communication do older adults experience themselves while using a robot to talk to others? What does that do for their feeling of 
authenticity? Will they 'hide' behind the robot? Will people transgress communicative norms because they can put the blame on a device?

To what degree will older adults perceive their robots as genuine social actors and personal representatives? Will they feel the presence of another human being through the presence of the social robot, for example, when the human conversation partner (B) and the user's personal robot ( $\mathrm{A}^{\prime}$ ) cooperate in coaching the user ( $\left.\mathrm{A}\right)$ ?

What would it be like if the teleconference is enriched with video, something the Pepper robot offers with its torso tablet (cf. [87])? Does it feel as if the robot takes over some of the physical representation of the distant other? Will older adults feel the presence of a social actor when no human is around and the robot displays some form of humanness? Is that different if such a robot is merely televised? What if a social robot takes care of business on their behalves, autonomously? Do people assume their psychological presence in a humanoid machine that takes their place?

One step further is how in human-humanoid robot interaction do we design self-directed robot-based education for older adults or let seniors follow robot-led virtual classes (cf. [88])? Will a robot representative feel like an alter ego (cf. Second Life avatars)? If two robot representatives make out, will that feel as cheating (cf. [89])?

General HRI explores the interaction with robots, including those that are not modeled after humans. In HHRI, the advantage is that the humanoid activates scripts of social conduct but not to the extent that humans expect the robot to be fully competent. This provides HHRI with a valuable social 'niche in the middle' [47]. Humanoids provide enough human cues to motivate people into behaviors that are social: the humanoid may assume the role of assistant or consultant and people may feel more resilient and less lonely because of it. Unlike humans, however, humanoids are forgiven if task execution is imperfect, when things come out the wrong way or misunderstandings occur [47]. Humanoids can be used to help people in a natural and intuitive way, yet because they are robots users may find mistaken human conduct funny before it becomes annoying.

Communication in HHRI usually looks at similarity between robot and human to find common ground in message transference. However, there is a positive side to otherness. Roboticists and designers may want to build in features that deliberately differ from face-to-face communication (e.g., LEDs for eyes, speaker ears, and camera mouth), not just for technical reasons but also to downplay the communicative skills of the robot, to lower expectations and hence render more positive net effects of conversational satisfaction.

Therefore, a robot as intermediary that brings people into contact as exemplified by the Call-me-Robby! proposal may be an effective new interaction paradigm in HHRI. Older adults may find it acceptable that the robot acts as the matchmaker but by the time its therapeutic capacities (i.e., $P A R)$ are exhausted, it is the other human being that may take over the conversation. As such, a humanoid makes an excellent conversation piece.

The effectiveness of the applications and designs in human-humanoid robot interaction and the relevance to our understanding of human behavior all depend on how a humanoid robot communicates and interacts or what could or perhaps should be different from human behavior. These are the new questions of HHRI with respect to artificial relationship formation, once our social robots are welcomed to our lives.

\section{Conflicts of Interest}

The author declares that there are no conflicts of interest regarding the publication of this paper.

\section{Acknowledgments}

The author thanks Marco Last for reviewing the initial draft of this paper. This publication was supported by the Institute of Social Resilience of the Vrije Universiteit [ISR-Call-2016Hoorn].

\section{References}

[1] C. Haub, World Population Aging: Clocks Illustrate Growth in Population under Age 5 and over Age 65, Population Reference Bureau, Washington, DC, USA, 2011.

[2] T. J. Holwerda, T. G. Van Tilburg, D. J. H. Deeg et al., "Impact of loneliness and depression on mortality: results from the Longitudinal Ageing Study Amsterdam," The British Journal of Psychiatry, Article ID 168005, pp. 127-134, 2016.

[3] L. C. Hawkley and J. T. Cacioppo, "Aging and loneliness downhill quickly?" Current Directions in Psychological Science, vol. 16, no. 4, pp. 187-191, 2007.

[4] W. Fisher, "Loneliness and sexuality", in Addressing Loneliness: Coping, Prevention And Clinical Interventions, A. Shaked and A. Rokach, Eds., pp. 34-50, Routledge, New York, NY, USA, 2015.

[5] E. C. Schoenmakers, T. G. van Tilburg, and T. Fokkema, "Problem-focused and emotion-focused coping options and loneliness: how are they related?" European Journal of Ageing, vol. 12, no. 2, pp. 153-161, 2015.

[6] Medical Advisory Secretariat, Social Isolation in CommunityDwelling Seniors: An Evidence-Based Analysis. Ontario Health Technology Assessment Series, Medical Advisory Secretariat, 2008.

[7] L. C. Hawkley, "Loneliness and social embeddedness in old age," in Encyclopedia of Geropsychology, A. N. Pachana, Ed., Springer, 2015.

[8] A. Koumpis, D. Casey, K. Murphy, and C. Kouroupetroglou, "The perceptions of people with dementia on robot companions and their potential to reduce loneliness and isolation," in Proceedings of the 25th Alzheimer Europe Conference Dementia: Putting Strategies and Research into Practice, H. V. LützauHohlbein and Š. Lukič Zlobec, Eds., p. 2, Ljubljana, Slovenia, 2015.

[9] S. Frennert and B. Östlund, "Review: seven matters of concern of social robots and older people," International Journal of Social Robotics, vol. 6, no. 2, pp. 299-310, 2014. 
[10] R. F. Baumeister and M. R. Leary, "The need to belong: desire for interpersonal attachments as a fundamental human motivation," Psychological Bulletin, vol. 117, no. 3, pp. 497-529, 1995.

[11] L. Campbell-Sills, D. H. Barlow, T. A. Brown, and S. G. Hofmann, "Effects of suppression and acceptance on emotional responses of individuals with anxiety and mood disorders," Behaviour Research and Therapy, vol. 44, no. 9, pp. 1251-1263, 2006.

[12] J. De Jong Gierveld and J. T. G. Van Tilburg, "Social Isolation and Loneliness," in Encyclopedia of Mental Health, H. S. Friedman, Ed., pp. 175-178, Academic Press, Oxford, UK, 2016.

[13] G. A. Bonanno, S. Galea, A. Bucciarelli, and D. Vlahov, "What predicts psychological resilience after disaster? The role of demographics, resources, and life stress," Journal of Consulting and Clinical Psychology, vol. 75, no. 5, pp. 671-682, 2007.

[14] M. T. Jacobs, van. Broese Groenou, M. I, A. H. De Boer, and D. J. H. Deeg, "Individual determinants of task division in older adults mixed care networks," Health and Social Care in the Community, vol. 22, no. 1, pp. 57-66, 2014.

[15] W. J. Chopik, "The Benefits of Social Technology Use among Older Adults Are Mediated by Reduced Loneliness," Cyberpsychology, Behavior, and Social Networking, vol. 19, no. 9, pp. 551556, 2016.

[16] J. Cohen-Mansfield, H. Hazan, Y. Lerman, and V. Shalom, "Correlates and predictors of loneliness in older-adults: a review of quantitative results informed by qualitative insights," in Proceedings of the International Psychogeriatrics, vol. 28, pp. 557576, 2016.

[17] American Psychological Association, The Road to Resilience, American Psychological Association, Washington, DC, USA, 2014.

[18] R. H. Pietrzak and S. M. Southwick, "Psychological resilience in OEF-OIF Veterans: Application of a novel classification approach and examination of demographic and psychosocial correlates," Journal of Affective Disorders, vol. 133, no. 3, pp. 560568, 2011.

[19] C. Panter-Brick and M. Eggerman, "Understanding culture, resilience, and mental health: The production of hope," The Social Ecology of Resilience: A Handbook of Theory and Practice, pp. 369-386, 2012.

[20] J. Kim-Cohen and R. Turkewitz, "Resilience and measured gene-environment interactions," Development and Psychopathology, vol. 24, no. 4, pp. 1297-1306, 2012.

[21] R. Yehuda, N. P. Daskalakis, F. Desarnaud, I. Makotkine, A. L. Lehrner, E. Koch et al., "Epigenetic biomarkers as predictors and correlates of symptom improvement following psychotherapy in combat veterans with PTSD," Frontiers in Psychiatry, vol. 4, p. 118, 2013.

[22] K. Sherrieb, F. H. Norris, and S. Galea, "Measuring Capacities for Community Resilience," Social Indicators Research, vol. 99, no. 2, pp. 227-247, 2010.

[23] G. A. Bonanno, A. Papa, K. Lalande, M. Westphal, and K. Coifman, "The importance of being flexible: The ability to both enhance and suppress emotional expression predicts long-term adjustment," Psychological Science, vol. 15, no. 7, pp. 482-487, 2004.

[24] C. Panter-Brick, "Health, risk, and resilience: Interdisciplinary concepts and applications," Annual Review of Anthropology, vol. 43, pp. 431-448, 2014.
[25] A. Ager, J. Annan, and C. Panter-Brick, Resilience From conceptualization to effective intervention. Policy Brief for Humanitarian and Development Agencies, 2013.

[26] A. S. Masten, Ordinary Magic: Resilience in Development, Guilford, New York, NY, USA, 2014.

[27] B. Thomlison, Family Assessment Handbook: An Introductory Practice Guide to Family Assessment, Brooks/Cole, Belmont, CA, USA, 3rd edition, 2010.

[28] S. E. Hobfoll, P. Watson, C. C. Bell et al., "Five essential elements of immediate and mid-term mass trauma intervention: empirical evidence," Psychiatry, vol. 70, no. 4, pp. 283-315, 2007.

[29] K. B. Adams, S. Sanders, and E. A. Auth, "Loneliness and depression in independent living retirement communities: risk and resilience factors," Aging \& Mental Health, vol. 8, no. 6, Article ID 13607860410001725054, pp. 475-485, 2004.

[30] E. L. Deci and R. M. Ryan, "Self-determination theory and the facilitation of intrinsic motivation, social development, and well-being," American Psychologist (Salma), vol. 55, no. 1, pp. 68-78, 2000.

[31] E. McIntyre, K. K. K. Wiener, and A. J. Saliba, "Compulsive Internet use and relations between social connectedness, and introversion," Computers in Human Behavior, vol. 48, pp. 569$574,2015$.

[32] WHO, World Health Statistics, World Health Statistics, Geneva, SW, 2012, http://apps.who.int/iris/bitstream/10665/44844/1/ 9789241564441_eng.pdf.

[33] G. Hofstede, Culture's consequences: Comparing Values, Behaviors, Institutions and Organizations across Nations, Thousand Oaks, CA, USA, 2001.

[34] K. Donnelly and J. M. Twenge, "Masculine and feminine traits on the Bem Sex-Role Inventory, 1993-2012: a crosstemporal meta-analysis," Sex Roles, pp. 0360-0025, 1-10, 2016, http://garote.bdmonkeys.net/bsri.html.

[35] J. Broekens, M. Heerink, and H. Rosendal, "Assistive social robots in elderly care: a review," Gerontechnology, vol. 8, no. 2, pp. 94-103, 2009.

[36] K. Wada, T. Shibata, T. Saito, and K. Tanie, "Effects of three months robot assisted activity to depression of elderly people who stay at a health service facility for the aged," in Proceedings of the In SICE, 2004, vol. 3, pp. 2709-2714, 2004.

[37] M. R. Banks, L. M. Willoughby, and W. A. Banks, "AnimalAssisted Therapy and Loneliness in Nursing Homes: Use of Robotic versus Living Dogs," Journal of the American Medical Directors Association, vol. 9, no. 3, pp. 173-177, 2008.

[38] C. C. Bennett, S. Sabanovic, J. A. Piatt, S. Nagata, L. Eldridge, and N. Randall, "A Robot a Day Keeps the Blues Away," in Proceedings of the 5th IEEE International Conference on Healthcare Informatics, ICHI 2017, pp. 536-540, USA, August 2017.

[39] H. Robinson, B. MacDonald, N. Kerse, and E. Broadbent, "The Psychosocial Effects of a Companion Robot: A Randomized Controlled Trial," Journal of the American Medical Directors Association, vol. 14, no. 9, pp. 661-667, 2013.

[40] R. Yu, E. Hui, J. Lee et al., "Use of a therapeutic, socially assistive pet robot (PARO) in improving mood and stimulating social interaction and communication for people with dementia: Study protocol for a randomized controlled trial," JMIR Research Protocols, vol. 4, no. 2, p. e45, 2015.

[41] K. Dautenhahn, A. Campbell, and D. S. Syrdal, "Does anyone want to talk to me?: Reflections on the use of assistance and companion robots in care homes," in Proceedings of the 4th 
Int Symposium on New Frontiers in Human-Robot Interaction, The Society for the Study of Artificial Intelligence and the Simulation of Behaviour (AISB), 2015.

[42] S. Parsons, A.-W. Kruijt, and E. Fox, "A cognitive model of psychological resilience," Journal of Experimental Psychopathology, vol. 7, no. 3, Article ID 053415, pp. 296-310, 2016.

[43] J. T. Fokkema and J. De Jong Gierveld, "Strategies to prevent loneliness," in Addressing Loneliness. Coping, Prevention and Clinical Interventions, A. Shaked and A. Rokach, Eds., pp. 218230, Routledge, New York, NY, USA, 2015.

[44] N. B. Raut, S. Singh, A. A. Subramanyam, C. Pinto, R. M. Kamath, and S. Shanker, "Study of loneliness, depression and coping mechanisms in elderly," Journal of Geriatric Mental Health, vol. 1, no. 1, pp. 20-27, 2014.

[45] R. S. Aylett, S. Louchart, J. Dias, A. Paiva, and M. Vala, "FearNot! - An Experiment in Emergent Narrative," in Intelligent Virtual Agents, vol. 3661 of Lecture Notes in Computer Science, pp. 305316, Springer Berlin Heidelberg, Berlin, Heidelberg, 2005.

[46] R. Aylett, J. Dias, and A. Paiva, "An affectively driven planner for synthetic characters," in Proceedings of the ICAPS 2006 - 16th International Conference on Automated Planning and Scheduling, pp. 2-10, UK, June 2006.

[47] J. F. Hoorn, E. A. Konijn, D. M. Germans, S. Burger, and A. Munneke, "The in-between machine: The unique value proposition of a robot or why we are modelling the wrong things," in Proceedings of the 7th International Conference on Agents and Artificial Intelligence (ICAART), S. Loiseau, J. Filipe, B. Duval, J. van den, and Herik., Eds., pp. 464-469, Lisbon, Portugal, 2015.

[48] E. L. Deci and R. M. Ryan, "Autonomy and need satisfaction in close relationships: Relationships Motivation Theory," in Human Motivation and Interpersonal Relationships: Theory, Research, and Applications, N. Weinstein, Ed., pp. 53-73, Springer, Dordrecht, Netherlands, 2014.

[49] F. Biocca, C. Harms, and J. K. Burgoon, "Toward a more robust theory and measure of social presence: review and suggested criteria," Presence: Teleoperators and Virtual Environments, vol. 12, no. 5, pp. 456-480, 2003.

[50] F. Eyssel and N. Reich, "Loneliness makes the heart grow fonder (of robots): on the effects of loneliness on psychological anthropomorphism," in Proceedings of the 8th ACM/IEEE international Conference on Human-Robot Interaction, H. Kuzuoka, V. Evers, M. Imai, and J. Forlizzi, Eds., pp. 121-122, Tokyo, Japan, March 2013.

[51] P. R. Lowenthal, "The Evolution and Influence of Social Presence Theory on Online Learning," in Online Education and Adult Learning, Advances in Educational Technologies and Instructional Design, pp. 124-139, IGI Global, 2010.

[52] G. Makransky, L. Lilleholt, and A. Aaby, "Development and validation of the Multimodal Presence Scale for virtual reality environments: A confirmatory factor analysis and item response theory approach," Computers in Human Behavior, vol. 72, pp. 276-285, 2017.

[53] V. K. Ng and R. A. Cribbie, "Using the gamma generalized linear model for modeling continuous, skewed and heteroscedastic outcomes in psychology," Current Psychology, pp. 1-11, 2016.

[54] M. Anderson and A. Perrin, Tech Adoption Climbs among Older Adults, Pew Research Center, Washington, DC, USA, 2017.

[55] M. Sánchez-Valle, M. Vinarás Abad, and C. Llorente-Barroso, "Empowering the elderly and promoting active ageing through the ternet: The benefit of e-inclusion programmes," in Safe at
Home with Assistive Technology, I. and Kollak., Eds., pp. 95-108, Springer, New York, NY, USA, 2017.

[56] M. B. Happ, "Mixed methods in gerontological research." Research in Gerontological Nursing, vol. 2, no. 2, pp. 122-127, 2009.

[57] R. Harris and E. Dyson, "Recruitment of frail older people to research: Lessons learnt through experience," Journal of Advanced Nursing, vol. 36, no. 5, pp. 643-651, 2001.

[58] J. W. Creswell and V. L. Plano Clark, Designing and Conducting Mixed Methods Research, SAGE, Thousand Oaks, CA, USA, 2007.

[59] J. F. Hoorn, "Mechanical empathy seems too risky. Will policymakers transcend inertia and choose for robot care? The world needs it," in Robotics - Legal, Ethical and Socioeconomic Impacts, G. Dekoulis, Ed., pp. 113-131, Croatia, Rijeka, 2017, http://www.intechopen.com/articles/show/title/mechanicalempathy-seems-too-risky-will-policymakers-transcend-inertiaand-choose-for-robot-care-the.

[60] S. MacLeod, S. Musich, K. Hawkins, K. Alsgaard, and E. R. Wicker, "The impact of resilience among older adults," Geriatric Nursing, vol. 37, no. 4, pp. 266-272, 2016.

[61] R. A. Paauwe, J. F. Hoorn, E. A. Konijn, and D. V. Keyson, "Designing robot embodiments for social interaction: Affordances topple realism and aesthetics," International Journal of Social Robotics, vol. 7, no. 5, pp. 697-708, 2015.

[62] K. Richardson, An Anthropology of Robots and AI: Annihilation Anxiety and Machines, New York, NY, USA, 2015.

[63] M. Dautzenberg and J. Guijt, Digital Communication in Home Care, Expertisecentrum Mantelzorg, Utrecht, Netherlands, 2016.

[64] A. Tapus, M. J. Mataric, and M. J. Matarić, "Socially assistive robots: the link between personality, empathy, physiological signals, and task performance. In," in Proceedings of the Papers of the AAAI Spring Symposium: Emotion, Personality, and Social Behavior, C. Lisetti, E. Hudlicka, I. Horswill, J. D. Velßsquez, and J. D. Velásquez, Eds., pp. 133-140, Menlo Park, CA, 2008.

[65] K. M. Lee, W. Peng, S.-A. Jin, and C. Yan, "Can robots manifest personality?: An empirical test of personality recognition, social responses, and social presence in human-robot interaction," Journal of Communication, vol. 56, no. 4, pp. 754-772, 2006.

[66] M. Nicolaisen and K. Thorsen, "What Are Friends for? Friendships and Loneliness over the Lifespan - From 18 to 79 Years," International Journal of Aging and Human Development, vol. 84, no. 2, pp. 126-158, 2017.

[67] N. L. Stevens and T. G. van Tilburg, "Cohort differences in having and retaining friends in personal networks in later life," Journal of Social and Personal Relationships, vol. 28, no. 1, pp. 24-43, 2011.

[68] M. M. Ernst and M. W. Mellon, "Acceptance and commitment therapy (ACT) to foster resilience in pediatric chronic illness," in Child and Adolescent Resilience within Medical Contexts. Integrating Research and Practice (Part 3), C. DeMichelis and M. Ferrari, Eds., pp. 193-207, Springer International.

[69] J. J. Gross, "Emotion regulation," in Handbook of Emotions, M. Lewis, J. M. Haviland-Jones, and L. Feldman Barrett, Eds., pp. 497-512, Guilford, New York, NY, USA, 3rd edition, 2008.

[70] D. A. Dillman, J. D. Smyth, and L. M. Christian, Internet, Phone, Mail, and Mixed-Mode Surveys: The Tailored Design Method, Wiley, Hoboken, NJ, USA, 2014.

[71] S. B. G. Eysenck, H. J. Eysenck, and P. Barrett, "A revised version of the psychoticism scale," Personality and Individual Differences, vol. 6, no. 1, pp. 21-29, 1985. 
[72] C. S. Carver, M. F. Scheier, and K. J. Weintraub, "Assessing coping strategies: a theoretically based approach," Journal of Personality and Social Psychology, vol. 56, no. 2, pp. 267-283, 1989.

[73] A. Aron, E. N. Aron, and D. Smollan, "Inclusion of Other in the Self Scale and the Structure of Interpersonal Closeness," Journal of Personality and Social Psychology, vol. 63, no. 4, pp. 596-612, 1992.

[74] E. Berscheid, M. Snyder, and A. M. Omoto, “The Relationship Closeness Inventory: Assessing the Closeness of Interpersonal Relationships," Journal of Personality and Social Psychology, vol. 57, no. 5, pp. 792-807, 1989.

[75] K. B. Starzyk, R. R. Holden, L. R. Fabrigar, and T. K. MacDonald, "The personal acquaintance measure: A tool for appraising one's acquaintance with any person," Journal of Personality and Social Psychology, vol. 90, no. 5, pp. 833-847, 2006.

[76] J. J. Scalise, E. J. Ginter, and L. H. Gerstein, "A Multidimensional Loneliness Measure: The Loneliness Rating Scale (LRS)," Journal of Personality Assessment, vol. 48, no. 5, pp. 525-530, 1984.

[77] J. de Jong Gierveld and T. van Tilburg, "The De Jong Gierveld short scales for emotional and social loneliness: Tested on data from 7 countries in the UN generations and gender surveys," European Journal of Ageing, vol. 7, no. 2, pp. 121-130, 2010.

[78] N. Schmidt and V. Sermat, "Measuring loneliness in different relationships," Journal of Personality and Social Psychology, vol. 44, no. 5, pp. 1038-1047, 1983.

[79] D. Russell, C. E. Cutrona, J. Rose, and K. Yurko, "Social and emotional loneliness: An examination of Weiss's typology of loneliness," Journal of Personality and Social Psychology, vol. 46, no. 6, pp. 1313-1321, 1984.

[80] G. Windle, K. M. Bennett, and J. Noyes, "A methodological review of resilience measurement scales," Health and Quality of Life Outcomes, vol. 9, no. 8, pp. 18-10, 2011.

[81] A. F. Hayes, Introduction to Mediation, Moderation, and Conditional Process Analysis: A Regression-Based Approach, Guilford, New York, NY, USA, 2013.

[82] A. P. Dickens, S. H. Richards, C. J. Greaves, and J. L. Campbell, "Interventions targeting social isolation in older people: a systematic review," BMC Public Health, vol. 11, 2011.

[83] C. Gardiner, G. Geldenhuys, and M. Gott, "Interventions to reduce social isolation and loneliness among older people: an integrative review," Health Social Care in the Community, pp. 1110, 2016.

[84] M. Choi, S. Kong, and D. Jung, "Computer and internet interventions for loneliness and depression in older adults: a meta-analysis," Healthcare Informatics Research, vol. 18, no. 3, pp. 191-198, 2012.

[85] M. Cattan, M. White, J. Bond, and A. Learmouth, "Preventing social isolation and loneliness among older people: a systematic review of health promotion interventions," Ageing and Society, vol. 25, Article ID S0144686, pp. 41-67, 2005.

[86] C. M. Masi, H.-Y. Chen, L. C. Hawkley, and J. T. Cacioppo, "A meta-analysis of interventions to reduce loneliness," Personality and Social Psychology Review, vol. 15, no. 3, Article ID 1088868310377394, pp. 219-266, 2011.

[87] S. O. Adalgeirsson and C. Breazeal, "MeBot: a robotic platform for socially embodied presence," in Proceedings of the 5th ACM/IEEE international Conference on Human-robot Interaction (HRI 10), P. Hinds, H. Ishiguro, T. Kanda, and P. Kahn, Eds., pp. 15-22, Osaka, Japan, March 2010.
[88] H. B. Vošner, S. Bobek, P. Kokol, and M. J. Krečič, "Attitudes of active older Internet users towards online social networking," Computers in Human Behavior, vol. 55, pp. 230-241, 2016.

[89] M. Scheutz and T. Arnold, "Are we ready for sex robots?" in Proceedings of the 11th ACM/IEEE International Conference on Human Robot Interaction (HRI '16), C. Bartneck, Y. Nagai, A. Paiva, S. abanovic, and S. Šabanović, Eds., pp. 351-358, Christchurch, New Zealand, 2016. 


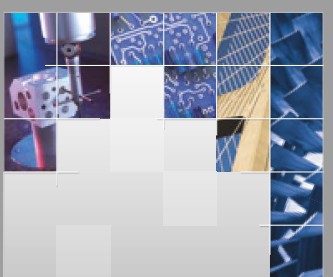

\section{Enfincering}
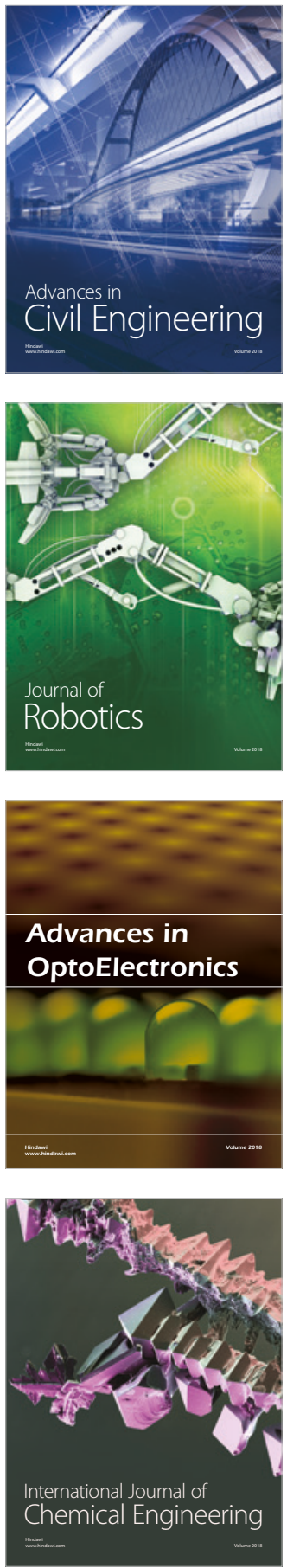

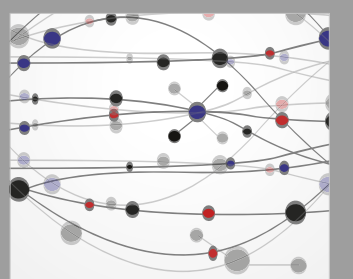

\section{Rotating \\ Machinery}

The Scientific World Journal

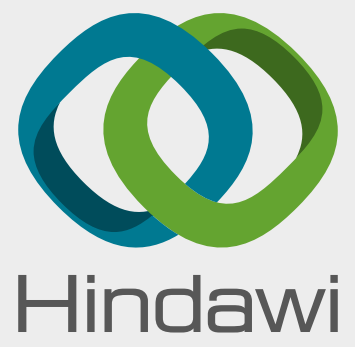

Submit your manuscripts at

www.hindawi.com
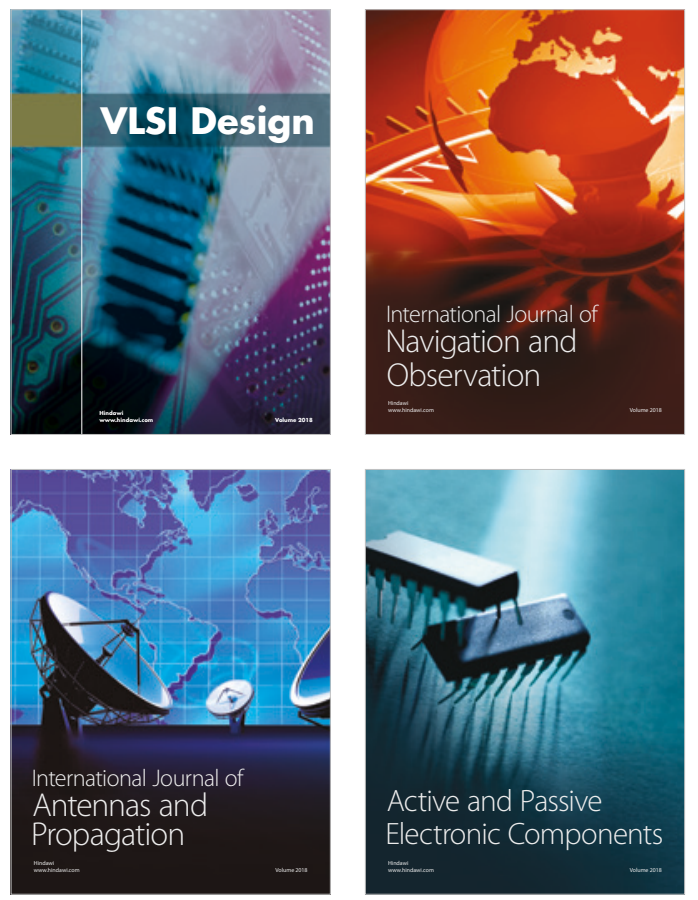
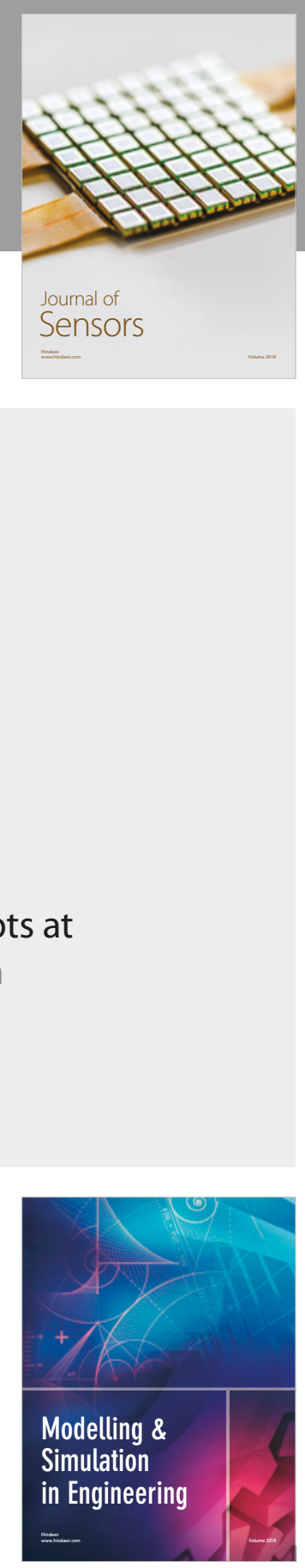

\section{Advances \\ Multimedia}
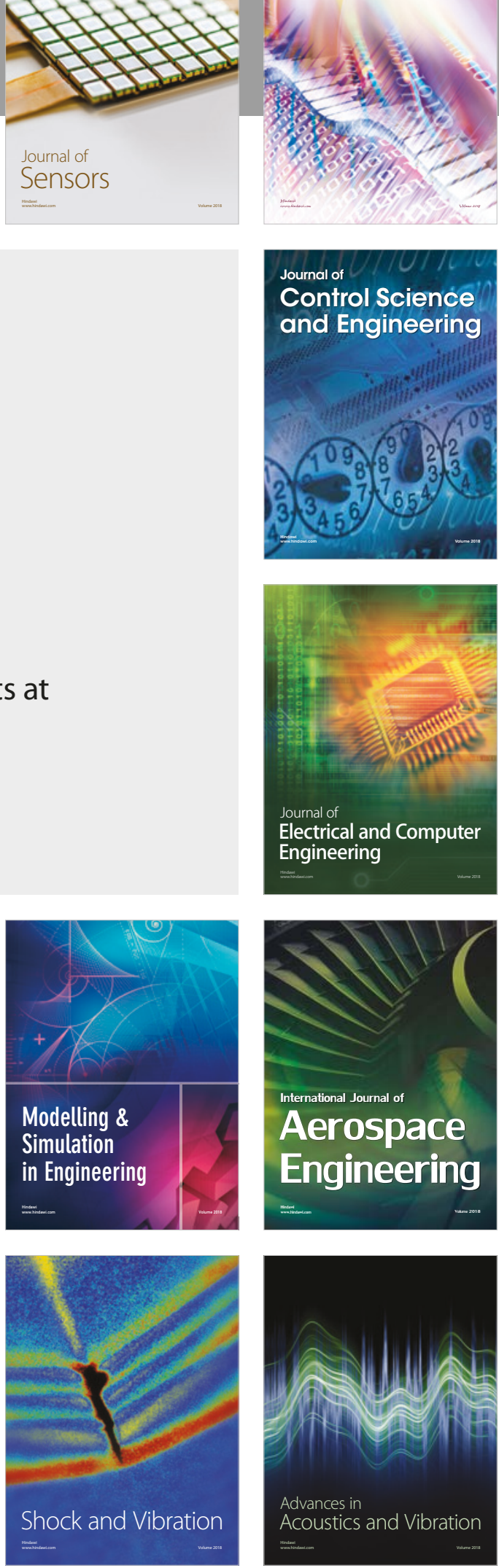